
du F.L.E. (18e et 19e siècles)

\title{
Le fait littéraire dans les manuels de français pour Espagnols au $18^{\mathrm{e}}$ siècle
}

\author{
Manuel Bruña Cuevas
}

\section{(2) OpenEdition Journals}

Édition électronique

URL : https://journals.openedition.org/dhfles/3011

DOI : $10.4000 /$ dhfles.3011

ISSN : 2221-4038

Éditeur

Société Internationale pour l'Histoire du Français Langue Étrangère ou Seconde

Édition imprimée

Date de publication : 1 décembre 1999

Pagination : p.95-115

ISSN : 0992-7654

Référence électronique

Manuel Bruña Cuevas, "Le fait littéraire dans les manuels de français pour Espagnols au $18^{e}$ siècle ", Documents pour I'histoire du français langue étrangère ou seconde [En ligne], 24 | 1999, mis en ligne le 18 janvier 2015, consulté le 27 mai 2021. URL : http://journals.openedition.org/dhfles/3011 ; DOI : https://doi.org/10.4000/dhfles.3011

Ce document a été généré automatiquement le 27 mai 2021.

(c) SIHFLES 


\title{
Le fait littéraire dans les manuels de français pour Espagnols au $18^{\mathrm{e}}$ siècle
}

\author{
Manuel Bruña Cuevas
}

1 Au $18^{\mathrm{e}}$ siècle, l'enseignement du français, comme celui des autres langues vivantes, a été peu porté sur tout ce qui avait trait à la littérature. Même les établissements innovateurs, ceux qui incorporaient dans leur plan d'études quelques disciplines scientifiques et quelque langue vivante, envisageaient celle-ci comme un instrument permettant l'accès à la production écrite dans la langue en question, mais à une production comprise au sens large, non restreinte au domaine littéraire; par là même, ils excluaient, eux aussi, toute introduction à la littérature d'une langue étrangère.

2 Ce n'est pas non plus dans les méthodes destinées au grand public pour apprendre le français que l'on peut trouver une attitude différente ${ }^{1}$. Malgré le fait que c'est justement le $18^{\mathrm{e}}$ siècle qui établit, aussi bien en France qu'en Espagne, un premier ensemble d'écrivains considérés comme des classiques de la langue vernaculaire, les méthodes de français pour Espagnols n'incluent généralement pas de morceaux choisis, d'échantillons de textes littéraires français pouvant servir de modèle pour le perfectionnement des capacités expressives de l'apprenant ${ }^{2}$. On est donc encore loin de ce qui sera une pratique courante aux $19^{\mathrm{e}}$ et $20^{\mathrm{e}}$ siècles, lorsque l'étude du français fera partie des plans d'études de l'enseignement secondaire et que les chrestomathies littéraires, incorporées ou non dans les grammaires, seront monnaie courante.

3 Le début du siècle semblait pourtant annoncer une situation toute différente. Dans ses premières années, deux grammaires de français pour Espagnols incluaient les règles de la versification française: celle de Pierre-Paul Billet (1708:309-352) et celle de l'abbé de Vayrac (1714:897-937). II est vrai, toutefois, que, malheureusement, ni l'un ni l'autre de ces auteurs ne fait œuvre originale; tous deux se bornent à traduire en espagnol des traités poétiques déjà existants, quoique la manière de s'y prendre varie légèrement de l'un à l'autre.

4 L'abbé révèle honnêtement sa source dès le titre de son traité, où l'on peut lire qu'il a été «sacado de la Gramatica Francesa del Padre Buffier de la Compania de JESUS» [tiré de la Grammaire française du Père Buffier, de la Compagnie de Jésus] ${ }^{3}$. Cet aveu initial, 
dont Buffier lui-même ne lui saura gré qu'à moitié ${ }^{4}$, lui permet, en toute bonne conscience, de traduire littéralement son modèle ${ }^{5}$, aussi bien les textes explicatifs que les morceaux poétiques donnés en exemple. Cette fidélité est si complète que Vayrac nous surprend en traduisant aussi la phrase de l'original par laquelle Buffier annonçait la parution prochaine d'un traité de poétique plus complet ${ }^{6}$. Or Jean de Vayrac est l'auteur de quelques ouvrages historiques (Histoire de la révolution d'Espagne, Paris, 1718), du célèbre Etat présent d'Espagne (Paris, 1718), d'une Nouvelle Grammaire Espagnole (Paris, 1708), etc., mais que nous sachions, il n'a jamais donné à la presse un traité de poétique autre que celui dont nous parlons.

Billet ne fait pas preuve de la même honnêteté que Vayrac. Non seulement il ne nous renseigne pas sur la source qui lui a permis de composer son art poétique, mais il s'évertue même à brouiller un peu les pistes qui auraient permis de la deviner. Nous avons découvert, en effet, que son art poétique suit de près la «Brève instruction sur les règles de la Poésie Françoise» que Claude Lancelot a incorporée, à la suite d'un traité sur la poésie latine, dans sa Nouvelle méthode pour apprendre facilement la langue latine. Billet garde le même ordre d'exposition, la même distribution de la matière que Lancelot, quoiqu'en la soumettant à une certaine réduction qui n'empêche pas, toutefois, l'obtention d'un résultat final bref, clair et cohérent. Il ne s'agit donc pas d'une simple traduction littérale de son modèle, comme c'est le cas chez Vayrac, mais plutôt d'une traduction libre, d'une adaptation lui permettant d'introduire ou de supprimer par-ci par-là certaines nuances. Là où Lancelot affirme, par exemple, que le sonnet est la composition en vers la plus difficile, mais également la plus belle ${ }^{7}$, Billet se limite à en signaler la difficulté, mais non la beauté. Il s'efforce même de fournir des exemples différents de ceux qu'il trouve chez Lancelot, quoiqu'il soit vrai aussi que, lorsque cela arrive, il se borne en général à chercher dans l'œuvre citée par Lancelot un autre passage avec les mêmes caractéristiques que celui donné par ce dernier. Curieusement, l'innovation la plus importante à cet égard résulte du fait que Billet substitue à certaines citations faites par Lancelot ses propres compositions à lui, le plus souvent sous prétexte -faux, par conséquent- qu'il n'a pas trouvé d'exemple pertinent chez d'autres poètes. Il a recours en cinq occasions (1708: 304, 306, 315, 316, 318-319) à ce stratagème, le meilleur, sans doute, non seulement pour faire accroire qu'il fait œuvre originale, mais aussi pour se faire connaître en tant que poète. Il profite, en fait, de la dernière de ces occasions -les trois sonnets situés à la fin de l'art- pour nous faire part de son intention de publier un recueil de ses vers ${ }^{8}$. Nous doutons fort que cette publication ait jamais dépassé le seuil de l'intention, annoncée dès la première édition de sa grammaire en 1673; le fait que la phrase en question est toujours présente dans celle de 1708 -alors qu'un certain nombre de changements ont été introduits à chaque réédition- semble indiquer que Billet n'avait pas trouvé d'éditeur pour sa production poétique en français ou qu'il a finalement préféré consacrer ses efforts à la publication de ses vers latins ${ }^{9}$ ou d'autres ouvrages ${ }^{10}$.

Une fois leur source connue, on est forcé de se demander quelles sont les raisons avouées ou non- qui ont pu pousser Billet et Vayrac à inclure un complément de versification dans leur travail, c'est-à-dire pourquoi ils ont décidé d'emprunter un chemin qui ne sera plus fréquenté par leurs successeurs ni ne l'avait été par leurs prédécesseurs (Bruña Cuevas, 1996b).

7 Aussi bien l'un que l'autre ont l'air de penser que leurs règles peuvent servir, non seulement à savoir apprécier les œuvres poétiques écrites en français, but que leur 
public espagnol aurait pu, peut-être, atteindre ${ }^{11}$, mais aussi à apprendre l'art de les composer, ce qui nous semble déjà hors de portée pour ce même public ${ }^{12}$. C'est pourtant le sens qui semble se dégager du titre qu'ils donnent à cette partie de leur ouvrage:

«Arte Poético, oCompendio breve de la Poesía Francesa, vtili-ssimo para aprender àconocer los versos y à componerlos». [Art poétique ou Abrégé de la poésie française, très utile pour apprendre à connaître les vers et à les composer.] (Billet, 1708).

Tratado de la Poesía Francesa, en el qual el curioso hallara las Reglas mas acertadas para componer todo genero de Versos.[Traité de la poésie française, où le curieux trouvera les meilleures règles pour composer tout genre de vers.] (abbé de Vayrac, 1714).

Or, si l'abbé de Vayrac s'en tient là, le cas de Billet est autrement intéressant. Il ajoute à cette motivation une seconde raison pour justifier la présence de l'Arte Pœtico dans son ouvrage. Il l'ouvre par une brève mais curieuse introduction («Prœmio»: 309-311), qui ne se trouve pas dans son modèle (Lancelot), et où l'on peut lire les propos suivants:

La Prosa Francesa, no siendo (como algunos piensan) el vnico assumpto de la estimación, que todas las Naciones de Europa ha-zen de nuestro Idioma; y mereciendo nuestra Poesía gran parte de los aplausos, que vnanimemente le dan, me parece, no lograría cumplidamente mi intento, si (después de averme explayado bastantemente sobre las reglas que di de la primera) no ofreciera àla curiosidad, y al desengaño, los preceptos que propongo de esta postrera, que si la estimación es hija de el conocimiento, espero mudaran de dictamen aquellos, que sin fundamento, no hazen caso de nuestra Poesía, y que a vista de este breve Compendio, no solo la tendrán en mejor concepto, pero aun confessaràn (por mas que obre (a passion) que nuestros versos, en nada son inferiores à los de las primeras lenguas del Mundo. Y si (hasta aora) perdieron, en la opinion de muchos, por faciles, è intele-gibles, suplico à los que tuvieren este dictamen, se desengañen, y se persuadan à que los versos, para ser buenos, no han de tener dificultades, ni la obscuridad de Oráculos; y que, si (para merecer estimación, y aplausos) fuera preciso el que necesitaran de Comentarios, parece, que la palabra que Dios concedió a los hombres, para explicar sus conceptos, aun trasladada al papel, no lograra su fin principal. Y no me aleguen los Comentarios que vemos oy de los Poetas Latinos, y Griegos, pues no milita la misma razón, y con muy diferentes circunstancias los comentaron, ô yà por averse perdido con el dilatado curso de los años las ideas, y noticias de lo que trataron, ôyà por aver passado sus obras à Naciones, que hablauan diferente Idioma.

[La prose française ne devant pas être (comme certains le pensent) la seule matière à faire l'objet de l'estime que toutes les nations de l'Europe ont pour notre langue, car notre poésie mérite une grande partie de ces applaudissements, il me semble que je n'atteindrais pas tous mes objectifs si, après avoir largement expliqué les règles de la première, je ne proposais, pour satisfaire les curieux et pour détromper certains, les préceptes de cette dernière. Si l'estime naît de la connaissance, j'espère que ce bref précis fera changer d'avis tous ceux qui, sans fondement, ne font pas cas de notre poésie, de sorte que, non seulement ils l'apprécieront davantage, mais ils avoueront, malgré le caractère passionnel de cette question, que nos vers ne sont en rien inférieurs à ceux des premières langues du monde. Je prie ceux qui leur ont jusqu'à présent reproché d'être faciles et intelligibles de se détromper et de se convaincre qu'il n'est pas obligé que les vers, pour être bons, présentent des difficultés ou l'obscurité des oracles; si, pour être dignes d'estime et de louange, ils devaient exiger d'être commentés, il semblerait que la parole, quoique octroyée par Dieu aux hommes pour leur permettre d'expliquer leurs pensées, ne remplirait pas, fût-ce par écrit, sa fonction principale. Qu'on ne me rétorque d'ailleurs pas qu'on consacre aujourd'hui des commentaires aux poètes latins et grecs, car ce cas est tout différent; si on les a commentés, c'est parce que le temps avait rendu floues les 
idées et les informations dont ils traitaient ou parce que leurs œuvres étaient abordées par des Nations parlant une langue différente.] des Espagnols à considérer que seules la poésie italienne et la leur, parmi celles écrites en langue vernaculaire, étaient dignes d'estime. Il est également possible que, en faisant référence à la facilité de compréhension de la poésie française, il vise à l'opposer à la poésie baroque -conceptiste ou gongoriste- prédominante à ce moment-là en Espagne. Il n'est pas à exclure que Billet cherche par là à compenser la généralisation abusive sur les capacités de la poésie française que l'on pouvait faire à partir de l'affirmation avancée par Lancelot dans sa grammaire d'espagnol, selon laquelle le français, contrairement à l'italien ou l'espagnol, n'était pas apte à la composition de vers libres, sans rime ${ }^{13}$. L'une quelconque de ces possibilités -ou toutes ensemble- a pu convaincre Billet de la nécessité de défendre la qualité des vers français auprès de son public espagnol. Mais, étant donné la partie finale de la citation ci-dessus, nous croyons plus probable que, tout simplement, il se fait par là l'écho du grand débat engagé au $17^{\mathrm{e}}$ siècle en France (François, 1959: 351-352) entre ceux qui proclamaient la supériorité des lettres classiques sur la littérature française et les défenseurs de celle-ci, parmi lesquels semble se ranger Billet ${ }^{14}$.

Quoi qu'il en soit, et indépendamment des buts avoués, nous pensons que la raison principale pour laquelle Billet et Vayrac ont inclu dans leur grammaire un art de la versification tient tout simplement à la simple imitation de leur modèle. La méthode de l'abbé, très peu adaptée à ses destinataires espagnols, n'est pratiquement qu'une transposition de celles de Régnier-Desmarais et Buffier. Comme ce dernier avait inclu dans son ouvrage, en tant qu'«addition» à la grammaire ${ }^{15}$, un traité de versification, Jean de Vayrac a dû se croire obligé de faire de même, imitant ainsi, sur ce point aussi, fidèlement son modèle. Quant à Billet, outre que son penchant de poète devait le porter naturellement à traiter d'un sujet qui lui était cher, l'exemple donné par Lancelot a dû jouer pour lui le même rôle que celui de Buffier pour Vayrac. Lancelot, en effet, n'avait pas seulement ajouté un traité de versification latine à sa grammaire de latin, mais il avait inclut les règles de versification italiennes dans sa grammaire d'italien (Paris, 1660) et celles de l'espagnole dans sa grammaire d'espagnol (Paris, 1660), ce qui a dû amener Billet à faire de même dans la composition de sa méthode de français à l'usage des Espagnols. Mais alors que l'abbé de Vayrac en reste là, on perçoit chez Billet une évolution en ce qui concerne la justification du bien-fondé de l'incorporation d'un traité poétique dans sa grammaire.

Chez l'abbé de Vayrac, le Tratado est présenté comme un ajout à la grammaire; ce n'est qu'après avoir clos celle-ci par les mots «Fin del Arte Françes» que le traité de versification est introduit dans l'ouvrage. Chez Billet, la question ne se présente pas de façon très différente lors de la première édition de 1673, publiée sous le litre de Gramática Francesa; dividida en dos partes [...] con un Arte Poética, o breve compendio de la Poesía Francesa [Grammaire française, divisée en deux parties... avec un art poétique ou précis de la poésie française]; Y Arte Poética y apparaît donc, de même que chez Vayrac, comme un complément à la grammaire proprement dite. Or, dès l'édition de 1688, le titre devient celui de Gramática Francesa, dividida en tres partes [divisée en trois parties], dont la troisième est constituée par l'art poétique, devenu de la sorte, pour l'auteur, une partie aussi importante que les deux autres pour rendre compte de la grammaire du 
français. Il est vrai que cette conception, comme nous l'avons vu, était déjà plus ou moins exprimée dans le «Prœmio» de la première édition:

[...] me parece, no lograria cumplidamente mi intento, si (despues de averme explayado bastantemente sobre las reglas que di de la primera [la prose, c'est-à-dire la langue en général]) no ofreciera à la curiosidad, y al desengaño, los preceptos que propongo de esta postrera [la poésie] [...] [il me semble queje n'atteindrais pas tous mes objectifs si, après avoir largement expliqué les règles de la première [la prose, c'est-à-dire la langue en général], je ne proposais, pour satisfaire les curieux et pour détromper certains, les préceptes de cette dernière [la poésie].]

Mais le changement de titre en 1688 pourrait révéler une prise de conscience plus ferme du fait que les règles de versification devaient être quelque chose d'inhérent à l'exhaustivité de toute grammaire. Billet a dû y parvenir grâce aux réflexions sur le concept de grammaire qu'il a été obligé de se faire à l'occasion de la publication par Jean-Pierre Jaron d'une nouvelle méthode de français à l'usage des Espagnols, l'Arte nueuamente compuesto de la Lengua Francesa por la Española (1688). En effet, cette publication amène Billet à incorporer dans l'édition de son ouvrage parue la même année (1688) une «Dissertacion critica» contre Jaron, où il soutient les propos que voici sur le terme Arte du titre de l'ouvrage de Jaron:

Detuueme en la voz Arte, y sin passar à leer la Epístola Dedicatoria, la Aprobación, ni el Prologo quise especular, discurriendo velozmente por todos los capítulos, si tenia esta fabrica todas las partes de que se deue cóponer vn Arte, y que ofrecia el pomposo titulo de Arte de la lengua Francesa, y reconocí estaua reducida a los solos primeros rudimentos, sin tener Sintaxi, ô tratado de oración, que corresponda al libro 4. de la lengua Latina, sin copia de frases, ô expressiones selectas, tan vtiles como se sabe, à la ensenâça de las lenguas: y finalmente, sin tratado de Poesia, que corresponde al lib. 5. de Nebrija: circunstancias todas tan essen-ciales à vn Arte, y que le constituyen tal.

[Je me suis arrêté au terme Arte et, sans entamer la lecture de la dédicace, l'approbation ou la préface, j'ai décidé de voir, en feuilletant rapidement tous les chapitres, si cet ouvrage, au titre pompeux de Arte de la lengua Francesa, présentait toutes les parties dont un art doit être composé. Or j'ai constaté qu'il était réduit aux seuls premiers rudiments, car il y manquait la Syntaxe -ou traité de la proposition correspondant au livre 4 de la langue latine-, les tournures -si utiles, comme on le sait-, et un traité de poésie correspondant au livre 5 de Nebrija, bien que toutes ces parties soient essentielles pour la constitution d'un art.]

Comme on le voit, Billet cache toujours le nom de l'auteur qui lui a servi de source directe, celui de Lancelot, mais ses références argumentatives n'en sont pas moins solides aux yeux de son époque. Dès la seconde édition de 1485 (Colombo Airoldi, 1993: 97), le cinquième livre de la grammaire latine de Nebrija, les ïntroductiones latinae (1481), était effectivement consacré à la métrique (Ramajo Caho, 1987: 35). Cette grammaire, d'autre part, était connue sous le nom de Arte, non seulement parce que ce terme était généralement employé comme synonyme de grammaire latine ${ }^{16}$, mais aussi du fait qu'elle avait été éditée plusieurs fois, dès le début du $16^{\mathrm{e}}$ siècle, sous le titre de $A d$ artem litterariam introductiones (Cano Aguilar, 1993: 88). La transformation du titre de la grammaire de Billet dans l'édition de 1688 pourrait donc ne pas être fortuite ${ }^{17}$, mais bien le fruit d'une décision de l'auteur visant à établir nettement que sa méthode de français était la seule vraiment complète, du moment qu'elle comprenait aussi bien les règles de la langue courante (c'est-à-dire, pour lui, celles de la prose) que les règles de la langue poétique, les unes et les autres constituant ensemble, et sur un pied d'égalité, la totalité de la grammaire d'une langue. 
Il est à remarquer que, malgré tout, Billet n'a pas appelé Arte sa méthode, alors que l'abbé de Vayrac a bien eu recours à ce terme pour donner un titre à son ouvrage ${ }^{18}$. Nous ne savons pas si ce dernier attribuait au terme la même valeur que Billet, mais il est permis d'en douter, du moment que l'abbé ne cherche nulle part dans son ouvrage à en justifier le titre. Quant à Billet, le fait que sa grammaire est parfois parue (1687) sans le précis de versification pourrait bien justifier que l'auteur ait renoncé au terme pour garder toujours le même titre à son ouvrage; mais il nous semble plus probable que la raison s'en trouve, comme nous l'avons indiqué, dans le fait que l'auteur n'a eu de conscience claire de la structure idéale souhaitable pour une grammaire qu'une fois que la première édition de son œuvre était déjà parue et qu'elle était devenue célèbre, c'est-à-dire à un moment où il n'était plus pertinent de changer le titre de la méthode.

Signalons, avant de quitter ces deux auteurs, un fait important. Chacun des points traités dans leur précis de versification est illustré par une citation, qui peut ne comprendre qu'un seul vers (dans la première partie des explications), mais qui peut également présenter des compositions entières ou presque entières (lorsqu'il s'agit de donner des exemples des différentes strophes). Aussi l'ensemble constitue-t-il un petit recueil de poésie française qui facilitait aux apprenants espagnols du français un premier contact avec la littérature composée en cette langue, leur permettant, du même coup, de se familiariser avec les noms de quelques poètes. Il faut se rappeler, toutefois, que la grammaire latine de Lancelot date du milieu du $17^{\mathrm{e}}$ siècle; aussi les auteurs proposés par Billet à ses lecteurs du début du $18^{\mathrm{e}}$ siècle n'étaient-ils pas du tout représentatifs de la littérature française de son époque; ils l'étaient des dernières décennies $\mathrm{du} 16^{\mathrm{e}}$ siècle ou de la première moitié $\mathrm{du} 17^{\mathrm{e}}$ siècle. Leur présence était parfaitement justifiée chez Lancelot, mais, chez Billet, des auteurs tels que Bertaut, Malherbe, Régnier et même Voiture, Benserade ${ }^{19}$ et l'omniprésent évêque de Grasse (c'est-à-dire Godeau, chez qui Lancelot et Billet puisent la plupart des citations) occupent une place qui aurait dû échoir aux écrivains du règne de Louis XIV. Somme toute, les seuls extraits contemporains cités par Billet ne sont autres que ses propres vers, s'il est vrai qu'il en était réellement l'auteur.

16 Cela change un peu avec l'abbé de Vayrac. Étant donné la proximité temporelle de la publication de l'ouvrage qu'il traduit (celui de Buffier), il cite des auteurs encore vivants ou morts depuis peu de temps. Malheureusement les noms ne sont pas abondants. On apprend qu'il ne faut pas suivre l'exemple de Boileau (cité sous le nom de Despréaux) ou de Corneille (Sr. Cornelia) et que Fontenelle (Sr. de Fontenela) est le seul poète qui ait été capable de composer un bon sonnet (non cité). Pour le reste, on a droit à quelques odes de Houdard de la Motte et, pour terminer le Tratado, à un extrait des fables de Mlle Bernard, à qui Jean de Vayrac -c'est-à-dire Buffier- consacre un panégyrique où il la compare à d'autres femmes écrivains célèbres ${ }^{20}$ :

[...] la difunta Mademoisela Bernard. No puedo concluir este Tratado por un nombre mas capaz de despertar el gusto de la Poesia. Pues esta ingeniosa Poeta puede ser colocada en la categoría de las Escuden, y de las Deshoulieres. [...feu Mademoiselle Bernard. Je ne peux terminer ce Traité par un nom plus apte à éveiller le goût pour la poésie, car elle peut être placée dans la catégorie des Scudéry et des Deshoulières.]

Comme nous l'avons dit, Billet et l'abbé de Vayrac sont des cas isolés dans notre corpus. Il faudra attendre, pour trouver une nouvelle référence à la versification française, l'édition, en 1815, de la grammaire de Monfort. Cet auteur complète le chapitre sur la prosodie -consacré au rythme et à la bonne prononciation de la langue en général- avec 
un point intitulé «Versificación». Son étendue -moins de deux pages (15-17)- et le fait qu'il est à son tour distribué en trois points différents -«Metro», «Rima» et «Cesura»indique assez, toutefois, le caractère sommaire de cette nouvelle approche, la première après un siècle de silence.

Après la grammaire de l'abbé de Vayrac, les méthodes du $18^{\mathrm{e}}$ siècle, méconnues ou célèbres, n'incluent donc jamais de références littéraires. Le $18^{\mathrm{e}}$ siècle, comme nous l'avons signalé plus haut, pratique un enseignement instrumental des langues étrangères. Cet enseignement vise parfois la langue parlée, ce qui entraîne l'incorporation dans les méthodes de dialogues destinés à l'illustrer (Galmace, 1748); c'est là une réminiscence de la plus ancienne des traditions dans l'enseignement des langues étrangères: celle des colloques et dialogues ${ }^{21}$. D'autres méthodes, publiées plus spécialement pour un public ayant besoin du français pour des raisons commerciales, incluent des modèles de lettres (Contaut, 1763), pratique remontant également au $16^{\mathrm{e}}$ siècle, puisque déjà en vigueur chez Berlaimont (1558). Finalement, les méthodes qui se veulent avant tout un instrument pour accéder à la connaissance des œuvres écrites en français -des œuvres écrites en général, quel que soit le sujet dont elles s'occupent-, incluent des morceaux de textes à traduire, mais, de façon cohérente, elles ne montrent aucune préférence pour les textes littéraires; les morceaux de thème ou version peuvent, certes, présenter parfois un caractère littéraire, mais ils ne sont alors jamais majoritaires. Tel est le cas de la grammaire de Chantreau (1781), qui inclut, parmi plusieurs textes historiques, journalistiques ou anecdotiques proposés pour la traduction, un seul morceau littéraire en français, un extrait des Aventures de Télémaque de Fénelon ${ }^{22} 22$. Le cas le plus intéressant à cet égard est toutefois représenté dans notre corpus par la grammaire de Paul-François Rousseau (1754).

La méthode de Rousseau, comme l'indique son titre (Rudimentos), est assez succincte, raison pour laquelle elle n'inclut pas de morceaux à traduire. Toutefois, l'auteur s'étend largement sur la fonction de la traduction dans l'apprentissage du français. À ce sujet, la première des règles qu'il donne, relative au choix des livres, constitue une sorte de résumé explicatif des raisons qui ont amené les auteurs de grammaires du $18^{\mathrm{e}}$ siècle à exclure le fait littéraire de leur œuvre. D'après Rousseau, il est nuisible aux débutants d'aborder la traduction des meilleurs textes littéraires en prose, car trop compliqués; il faut donc exclure aussi, à plus forte raison, les textes en vers:

No todos los libros son buenos para un principiante, no entiendo solo los libros de Poesía, cuya inteligencia pueden apenas alcanzar los mas diestros, por las transposiciones, figuras, y otras licencias, que permite, y aun manda el Arte, sino también obras de prosa, como son las de los Señores Bossuet, Fènélon, Bellegarde, y Voltayre, y otras de esta classe, cuyo estilo es un compuesto de phrases las unas selectas, y tan proprias del Idioma, que quererlas traducir al pié de la letra (lo que es proprio de un principiante) seria, no solamente darle malos principios; pero también privarle de la prompta inteligencia de los libros, único afán suyo, y gloria del Maestro, que con ella cubrirá en algún modo lo mercenario de sus tareas.

[Tous les livres ne sont pas bons pour des débutants. J'entends par là non seulement des livres de poésie -que les plus doués ne parviennent guère à comprendre à cause des transpositions, figures et autres licences que l'Art- permet, et même conseille, mais aussi des œuvres en prose, telles que celles de Bossuet, Fénelon. Bellegarde, Voltaire et d'autres de ce genre, au style fondé sur l'emploi de tournures et de gallicismes, impossibles à traduire au pied de la lettre comme ont tendance à le faire les débutants. Leur proposer ces textes supposerait, non seulement leur enseigner de mauvais principes, mais encore les priver d'une compréhension immédiate des livres, alors que celle-ci est, d'une part, leur but principal et. de 
l'autre, la gloire du Maître, qui justifie par là, en quelque sorte, le côté mercenaire de sa tâche.] (1754: 62-63). les grammaires -du moins leur traités poétiques- lui auraient certainement paru tout à fait inadaptées aux besoins des débutants. Pour Rousseau, les textes proposés doivent être relativement faciles et présenter un intérêt pour les apprenants ${ }^{23}$; voici la suite $d u$ passage précédent:

Lograràse uno, y otro, tomando libros de estilo culto, pero familiar, y llano, como son libros de Historias; si se quiere, libros de la facultad del Discípulo, pero no ágenos de ella; porque si no los entendiera en su Idioma nativo, cómo los entenderá en ageno? Pueden hacer al caso las obras del Señor Bussi Rabutin, el Thèlèmaco, los Tratados del Padre Regnault, el de la Opinion, Cartas Familiares, las Historias de el Señor Rollin, \&c. cuyas dificultades, à lo mas, se pueden reducir al trabajo material de buscar muchos términos en el Vocabulario: exercicio sin duda muy util.

[On réussira l'une et l'autre de ces choses en choisissant des livres de style soigné, mais familier et facile; des livres d'histoires, par exemple, ou, si l'on veut, des livres portant sur des sujets familiers au disciple, car, incapable de les comprendre dans sa langue maternelle, comment les comprendrait-il dans une langue autre? On peut proposer les œuvres de Bussy-Rabutin, le Télémaque, les traités du père Regnault, celui de l'Opinion, des lettres familières, les Histoires de Rollin, etc., dont les difficultés se réduisent, tout au plus, au travail matériel de chercher beaucoup de termes dans le dictionnaire, exercice sans doute très utile.] (ibid.: 63).

21 Comme nous l'avons dit, c'est en quelque sorte ce même critère que suivra Chantreau dans son choix des morceaux à traduire. Mais Chantreau présenle un intérêt bien plus grand que Rousseau en ce qui concerne notre sujet. Nous venons de voir que ce dernier déconseille aux débutants la fréquentation de certains auteurs célèbres; mais il n'en fournit pas moins quelques noms importants dans l'histoire de la littérature française des $17^{\mathrm{e}}$ et $18^{\mathrm{e}}$ siècles, fait insolite depuis la parution de la grammaire de l'abbé de Vayrac. Or c'est à Chantreau que revient le mérite d'avoir recommandé, pour la première fois dans l'ensemble des œuvres de notre corpus, une liste variée de textes littéraires français.

Chantreau s'accorde avec Rousseau sur le critère qui doit présider au choix des textes à traduire. Ceux qu'il propose le prouvent suffisamment. Mais, en plus, il inclut dans les dernières pages de son ouvrage (1786: 304-308) -ce que Rousseau n'a pas fait- une «Bibliothèque Française ou choix des livres que tout amateur de la littérature doit se procurer». Le terme littérature y est toutefois employé dans le sens large de «textes écrits», de sorte que les livres relevant de ce que nous entendons par littérature ne représentent qu'un petit nombre dans l'ensemble constitué par des ouvrages de grammaire, logique, morale, mythologie, géographie, voyages, histoire (les mieux représentés: 22 titres), physique et histoire naturelle et tactique militaire. Les œuvres à caractère littéraire sont réparties en quatre groupes: «Littérature», «Poésie», «Théâtre» et «Romans, Contes». Le premier comprend des ouvrages de théorie littéraire ou de philosophie de grand impact au $18^{\mathrm{e}}$ siècle (Principes de Littérature de La Bléterie; Cours de Belles-Lettres de Batteux; Essai sur l'origine des connaissances humaines et Traité des sensations, de Condillac) et des recueils divers (Modèles de lettres sur différents sujets. Bibliothèque d'un homme de goût pour le choix des meilleurs livres). En ce qui concerne la poésie, il recommande Boileau, Racine fils (c'est-à-dire le poème d'inspiration janséniste, La Religion, 1742, de Louis Racine, fils du dramaturge) et le recueil Le Portefeuille d'un homme de goût. Parmi les œuvres dramatiques, on trouve celles de la

Documents pour l'histoire du français langue étrangère ou seconde, 24 | 1999 
triade classique, Corneille, Racine et Molière, mais aussi, pêle-mêle, celles d'autres auteurs des $17^{\mathrm{e}}$ et $18^{\mathrm{e}}$ siècles, aussi bien tragiques \{Le Comte $\mathrm{d}^{\prime} E s s e x$ de Thomas Corneille, les pièces de Crébillon père et Le Siège de Calais, 1765, de De Belloy) que comiques (les comédies de Regnard, La Métromanie de Piron, 1738, et Le Méchant de Gresset, 1745) ${ }^{24}$. Dans le dernier groupe, enfin, Chantreau inclut aussi quelque ouvrage du $17^{\mathrm{e}}$ siècle (les Fables de La Fontaine, curieusement) et, pour le $18^{\mathrm{e}}$, le célèbre Gil-Blas (1715-1735) et les Contes moraux de Marmontel, ainsi qu'un Dictionnaire d'Anecdotes.

Il est vrai qu'il manque à cette liste quelques noms d'écrivains que nous considérons aujourd'hui comme des classiques des $17^{\mathrm{e}}$ et $18^{\mathrm{e}}$ siècles ${ }^{25}$, mais l'effort fourni par l'auteur en vue de permettre aux lecteurs espagnols une approche de la littérature française n'en est pas moins considérable, notamment si l'on songe à l'absence d'intérêt pour le fait littéraire dans les grammaires précédentes.

Nous nous devons, avant de terminer, de rendre hommage à un ouvrage qui, quoique n'étant pas une grammaire, a dû être fréquemment consulté par bon nombre d'Espagnols s'intéressant au français; nous pensons au dictionnaire de Torre y Ocôn (1728). Notre hommage, pourtant, n'a pas comme destinataire l'auteur -qui ne souffle mot sur notre sujet ni dans cet ouvrage ni dans la grammaire dont il est également responsable (1728)-, mais l'un des censeurs chargés d'approuver la publication du dictionnaire: Fernando Trivino.

L'approbation rédigée par Trivino pour le dictionnaire de Torre y Ocôn trace une petite histoire des trois littératures en langue vulgaire les plus notables pour lui: l'italienne, l'espagnole et la française. Il commence par établir que la fortune d'une langue est fonction de la force militaire et de l'amour des lettres du peuple qui la parle, le français ayant conquis dernièrement une position privilégiée par l'un et l'autre de ces chemins: d'une part, les armées et les bâtiments militaires ou marchands de Louis XTV l'ont répandu aussi bien en Europe que dans tout le monde habité; de l'autre,

[...] las estudiosas, infatigables tarèas de tantos varones doctos como florecieron en Francia el Siglo passado, (al quai con razon llaman los Franceses, por lo que mira à las Letras, el de Oro, y el de Augusto) la han pulido, limado, y enriquecido con tantas sabias, eruditas, y corregidas Obras, que en cierto modo se haze preciso à los aficionados à las Artes, y las Ciencias, el aprender este idioma, para no verse privados de los opimos frutos de tan provechosas fatigas.

[...les efforts des nombreux hommes cultivés qui vécurent en France au siècle passé (appelé à bon droit par les Français, en ce qui concerne les lettres, le siècle d'or et le siècle d'Auguste) l'ont polie, épurée et enrichie d'un si grand nombre d'œuvres profitables, érudites et soignées que tout amateur des arts et des sciences est aujourd'hui obligé d'apprendre cette langue afin de ne pas être privé des excellents fruits de leur travail.]

Or cette place actuelle du français avait été précédemment occupée, tour à tour, par l'italien et par l'espagnol. L'étape de prédominance de l'italien s'est étendue de la fin du $14^{\mathrm{e}}$ siècle au début du $16^{\mathrm{e}}$, c'est-à-dire "desde el tiempo de Dante, Bocaccio, y Petrarca, hasta el de Torquato Tasso». L'espagnol, de son côté, a commencé sa course littéraire au $16^{\mathrm{e}}$ siècle, pour atteindre son plein essor au début $\mathrm{du} 17^{\mathrm{e}}$ siècle, le règne de Philippe II pouvant donc être considéré comme le siècle d'Or des lettres espagnoles. Cette perfection acquise, la décadence, selon Triviño, était inéluctable, la plupart des écrivains espagnols ayant pratiqué depuis un barroquisme exécrable. Or, c'est juste à ce moment que commence la splendeur des lettres françaises:

Finalmente entro en la sabia arena la Lengua Francesa à los principios del Siglo passado, y debiendo à vna dichosa casualidad el origen de su Academia, y sus 
adelantamientos à la protección de unMecenas purpurado, y Ministro poderoso, se fue poco a poco perfeccionando a impulsos de la liberalidad del Gran Luis, y de la virtuosa emulación de los literatos, de suerte que empezaron à florecer, y descollar vn Malherbe, dos Comelios, Balzac, Voiture, Moliere, Racine, Vaugelàs, Ménage, Régnier, Boileau, Flechier, Bourdaloué, y otros muchos Oradores, y Poetas de gran nombre, y fama; pues los vnos traduciendo con acierto los mas celebrados originales Griegos, y Latinos, y los otros imitando en sus escritos aquellos antiguos admirables modelos, enriquecieron la República Literaria, y ennoblecieron su patrio idioma.

[Enfin, au début du siècle dernier, ce fut à la langue française de faire sonentrée dans la savante arène. Devant l'origine de son Académie à une circonstance heureuse, et ses progrès à la protection d'un mécène pourpré, elle s'est peu à peu perfecüonnée grâce à l'élan généreux de Louis le Grand et à la saine émulation de ses écrivains, de sorte qu'on a vu apparaître un Malherbe, deux Corneille, Balzac, Voiture, Molière, Racine, Vaugelas, Ménage, Régnier, Boileau, Fléchier, Bourdaloue et beaucoup d'autres orateurs et poètes renommés; les uns en traduisant avec succès les originaux grecset latins les plus renommés, les autres en imitant dans leurs écrits ces admirables modèles anciens, ont enrichi la République littéraire et anobli leur langue.]

Il est surprenant que cet exemple n'ait pas encouragé les auteurs ou censeurs des dictionnaires et des grammaires postérieurs à consacrer quelques commentaires à l'importance de la littérature et des écrivains français. Nous avons exposé ci-dessus les causes qui peuvent l'expliquer, mais nous ajouterons encore, en ce qui concerne plus particulièrement les dictionnaires bilingues français-espagnol, que le fait qu'ils ont été le plus souvent publiés en France -visant donc en premier lieu un public français intéressé à l'étude de l'espagnol- rend compte aussi d'un tel état de choses. Il nous semble que la perte de prestige de l'espagnol et des lettres espagnoles en France pendant le $18^{\mathrm{e}}$ siècle (Guillén 1994:123) a amené les auteurs de ces dictionnaires à consacrer, dans les prologues, tous leurs efforts à défendre l'espagnol, ce qui a joué au détriment d'une exposition détaillée des valeurs du français et de sa littérature, réputées connues. C'est ainsi que Gattel, dans le long «Discours préliminaire» qui ouvre son dictionnaire bilingue (1790), insiste largement sur l'importance des lettres espagnoles, mais ne fait allusion aux lettres françaises que pour signaler qu'elles ont été très influencées au $17^{\mathrm{e}}$ siècle par les écrivains espagnols (il donne l'exemple du Cid de Corneille) ou pour rappeler que certains auteurs français -seuls Scarron et Rabelais sont mentionnés- avaient pratiqué, comme Quevedo en Espagne, l'invention de nombreux termes qui ne sont jamais passés dans la langue commune.

Plusieurs facteurs ont donc contribué à l'absence de références littéraires dans le corpus -grammaires ou dictionnaires- que nous avons consultés. Les exceptions que nous avons signalées n'en méritent que davantage toute notre estime. 


\section{BIBLIOGRAPHIE}

BILLET, Pierre-Paul (1703): Opusculorum Miscellaneorum Latino Sermone Scríptorum, Madrid, Manuel Ruiz de Murga, BN 3/3797.

BRUÑA Cuevas, Manuel (1996a): «La literatura en la enseñanza del francés en España en los siglos XVI al XVTII», Aproximaciones diversas al texto literario, éd. par J. Martínez, C. Palacios \& A. Saura, pp. 9-17, Murcia, Servicio de Publicaciones de la Universidad.

BRUÑA Cuevas, Manuel (1996b): «Le problème de l'orthographe française dans les grammaires de français à l'usage des Espagnols et dans les dictionnaires bilingues français-espagnol et espagnolfrançais (XVI ${ }^{\mathrm{e}}-\mathrm{XVIII}{ }^{\mathrm{e}}$ siècles)», La lingüística francesa: gramática, historia y epistemología, éd. par E. Alonso, M. Bruña y M. Muñoz, pp. 85-101, Séville, Grupo Andaluz de Pragmática.

BRUÑA Cuevas, Manuel (1998): «Présentation de l'universalité de la langue française dans les grammaires de français pour les Espagnols et dans les dictionnaires bilingues antérieurs à 1815», Historiographia Linguistica, 25 (sous presse).

BUFFIER, Claude (1723 [1709]): Grammaire Françoise sur un plan nouveau; Avec [...] un Abrégé des régies de la Poésie Françoise, Paris, N. Le Clerc. (La première édition est le n. ${ }^{\circ} 65$ des Archives de la Linguistique Française).

CANO AGUILAR, Rafael (1993): «Antonio de Nebrija y Mateo Alemán: los orígenes de la gramática en España y América», Anuario de Letras, 31, pp. 65-91.

CARAVOLAS, Jean-Antoine (1995): «Apprendre à parler une langue étrangère à la Renaissance», Historiographia Linguistica, 22/3, pp. 275-309.

COLOMBO AIROLDI, Fulvia (1993): «Nebrija, precursor de la enseñanza del español como lengua extranjera», Anuario de Letras, 31, pp. 93-120.

FRANÇOIS, Alexis (1959): Histoire de la langue française cultivée. Genève: A. Jullien.

GUILLEN, Claudio (1994): «Imágenes nacionales y literatura», Anales de literatura española, 10, pp. 117-Í45.

LANCELOT, Claude (1660): Nouvelle méthode pour apprendre facilement et en peu de temps la langue espagnole, Paris, Pierre Le Petit, Rééd. par E. Hernández \& M.I. López Martínez. Murcia, Servicio de Publicaciones de la Universidad.

LANCELOT, Claude (1761 [1644]): Nouvelle méthode pour apprendre facilement la Langue Latine; [...] Avec un Traité de la Poésie Latine, \& une breve instruction sur les Régies de la Poésie Françoise, Paris, Guillyn.

LÉPINETTE, Brigitte (1991): «Les Dialogues (1618) de César Oudin», Actas del Primer Coloquio Internacional de Traductología, éd. par B. Lépinette, M. a A. Olivares \& E. Sopeña, pp. 139-145, Valencia, Universität de Valencia.

LÉPINETTE, Brigitte (1995): «La grammaire constrastive franco-espagnole de la première moite du $\mathrm{XVIII}^{\mathrm{e}}$ siècle. Analyse de six ouvrages édités en Espagne», Historiographia Linguistica, 22/1-2, pp. 27-74.

LÉPINETTE, Brigitte (1996): «Les premières grammaires du français (1565-1799) publiées en Espagne. Modèles, sources et rôle de l'espagnol», Histoire, Épistémologie, Langage, 18/2, pp. 149-177. 
RAMAJO CAÑO, Antonio (1987): Las gramáticas de la lengua castellana desde Nebrija a Correas, Salamanque, Ediciones de la Universidad.

RÉGNIER-DESMARAIS, François-Séraphin (1706 [1705]): Traité de la grammaire française, Paris, JeanBaptiste Coignard. (Genève: Slatkine Reprints, 1973).

ROLDAN PÉREZ, Antonio (1976): «Motivaciones para el estudio del español en las gramáticas del siglo XVI», Revista de Filología Española, 58, pp. 201-229.

SÁNCHEZ, Aquilino (1987): «Renaissance méthodologies for teaching spanish as a foreign language», Histoire Épistémologie Langage, 9/2, pp. 41-60.

SWIGGERS, Pierre (1984): Les Conceptions linguistiques des Encyclopédistes. Étude sur la constitution d'une théorie de la grammaire au siècle des Lumières, Heidelberg, Julius Groos Verlag.

SWIGGERS, Pierre (1996): «Language contact, language history and history of linguistics: John Palsgrave's 'Anglo-French' grammar (1530)», La description du français au XVI siècle: deux études, pp. 1-13, Louvain, Département Linguistiek of the Katholieke Universiteit Leuven (n. $\left.{ }^{\circ} 155\right)$.

VAYRAC, Jean de (1718): Etat présent d'Espagne, Paris, Antonin des Hayes, BN 2/5614-7.

VAYRAC, Jean de (1714 [1709]): Nouvelle grammaire espagnole, Paris, Pierre Witte.BN 3/3391.

\section{NOTES}

1. Sur les différents types de grammaires de français pour Espagnols publiées en Espagne du 16' au $18^{\mathrm{e}}$ siècle, voir Lépinette $(1995,1996)$.

2. Aucun des ouvrages de notre corpus ne pratique non plus la méthode de Palsgrave, qui fournissait, pour illustrer ses explications grammaticales, outre ses propres exemples, des exemples contextualisés tirés d'un corpus d'auteurs (Swiggers, 1996: 3).

3. L'abrégé sur la poésie a été ajouté par Buffier à sa grammaire à partir de la deuxième édition de Paris (1714), qui doit être, par conséquent, celle qui a servi de modèle à Vayrac. Il ne se trouve pas encore dans l'édition de Bruxelles de 1711, faite, selon Buffier (préface de l'édition de 1714), sans son autorisation préalable.

4. Dans l' "Avertissement sur cette dernière Edition» (1723), Buffier, après s'être plaint que son ouvrage avait été critiqué ou saccagé par plusieurs auteurs, ajoute les phrases suivantes, faisant allusion, à notre avis, à la grammaire de Vayrac: «Un autre a pris encore des morceaux entiers de mon ouvrage qu'il a revêtus d'un langage Espagnol; mais lui du moins il l'avoue bonement, \& dit que c'est qu'il ne trouvoit rien de mieux. Peut-être néanmoins auroit-il encore été mieux, d'emprunter tout l'ouvrage pour le traduire en Espagnol, ou de citer les endroits qu'il empruntoit pour se métré plus à couvert du soupçon de Plagiaire.»

5. Littéralement mais non sans commettre certaines méprises. Là où Buffier dit, par exemple, que ia, ie, ieu, ieus, ieux ne font qu'une syllabe «dans les noms substantifs fort courts \& dans leurs dérivez» (1723:492), Vayrac donne la traduction suivante: «ia, ie, ieu, ieus, ô ieux, losquales en los Nombres Substantivos son muy brèves, y no hazen mas que una sylaba en sus derivados». [ia, ie... lesquels, dans les noms substantifs, sont très brefs et ne font qu'une syllabe dans leurs dérivés].

6. En parlant des œuvres de Mlle Bernard, l'abbé de Vayrac écrit: «Espero poner en una Obra mas amplia algunos otros partos de su ingenio del mismo gusto» (1714: 936). Voici le texte de Buffier: «J'espére insérer dans un ouvrage plus ample, quelques autres de ses pièces du même goût» (1723:524). Buffier fait d'ailleurs cette annonce à deux reprises dans son «Abrégé nouveau des règles de la pœsie françoise» (1723:486-525); à la fin du traité, par la phrase que nous venons de transcrire, et au début: «Je ne prétens pas ici parler de tout ce qui regarde en général la Poésie, 
ou même la Poésie Françoise: ce qui me demanderoit un Traité exprès que j'espére faire un jour à la suite d'un Traité d'éloquence.» (1723:486).

7. «Il n'y a gueres d'Ouvrages en Vers qui soient plus beaux que le Sonnet, ni aussi plus difficile.» (1761:814).

8. De vno, y otro servirán de exemplos los sonetos que se siguen, que ofrezco à la curiosidad de el Lector, entre tanto, que salen à luz las Pœsias, que para alivio, y descanso de estudios, y ocupaciones mas serias, ha producido à ratos mi Musa.» [Les sonnets suivants serviront d'exemple. Je les propose au lecteur en attendant la publication des poèmes que j'ai composés pour me reposer d'autres occupations plus sérieuses.] (1708:351).

9. Opusculorum Miscellaneorum Latino Sermone Scriptorum (Madrid, 1703).

10. Dans la préface de sa traduction, La verdad en su luz (Madrid, 1677), il donne le titre d'un ouvrage en préparation que nous n'avons pu trouver. Nous ne savons donc pas s'il devait traiter du même sujet que la traduction ou si c'est le titre qu'il pensait donner au recueil poétique en français qu'il avait annoncé quelques années plus tôt. Voici le texte de celte préface: «Recibe este fruto, aunque corto presente, aguardando à que (maduro el de los Sueños verdaderos, o Verdades soñadas, que te está previniendo mi cuydado) te lo ofrezca mi obsequio» [Reçois ce fruit, tout modeste qu'il soit, en attendant que je puisse t'offrir les Sueños verdaderos, à Verdades soñadas que je prépare actuellement.]

11. Voir plus loin, à cet égard, l'avis tout à fait contraire de Paul-François Rousseau.

12. Claude Lancelot, dans la préface de sa grammaire espagnole (1660), signale que les règles de versification qui l'accompagnent peuvent aider les débutants à aborder la lecture des poètes espagnols, mais ne fait pas allusion à la possibilité qu'elles puissent servir également à aider les Français à composer des vers espagnols.

13. Dans sa grammaire d'espagnol, Lancelot affirme: «Cela fait voir que la langue Espagnole, \& l'Italienne ont quelque chose dans le son \& l'arrangement des mots qui se soustient mieux que dans la nostre, puis qu'elles peuvent faire des vers qui contentent l'oreille, \& qui se fassent sentir pour vers, sans y employer des rimes; ce que nous ne sçaurions faire. [...] D'où vient que les vers Héroïques. Lyriques, \& autres qu'ils ont imité des Latins, ont esté quelquefois plus heureusement employez en leur langue qu'en la nostre, quoy qu'a-present ils s'en seruent peu.» 0660:121).

14. Le fait que Billet est l'auteur de vers latins n'est pas du tout contradictoire avec cette prise de position. Que l'on se rappelle que le chef de file du parti des Anciens n'était autre que Boileau.

15. Voici ce que dit Buffier à propos de ces additions dans la préface de la première édition: «Je donne à la troisième Partie le nom d'Additions; parce que les choses dont je l'ai remplie, quelque utiles qu'elles soient, peuvent être censées de surérogation dans une simple Grammaire.»

16. C'est une acception qu'on trouve encore dans le dictionnaire de l'Académie espagnole

17. Elle ne serait fortuite en aucun cas. Si nous n'affirmons pas de façon plus tranchée que ces explications sont la véritable cause de la transformation du titre, c'est tout simplement parce qu'il se peut que celle-ci ait été due, tout simplement, à des raisons commerciales, c'est-à-dire à l'habitude de présenter toute réédition d'un ouvrage comme ayant été «corrigé et augmenté» par rapport à l'édition précédente. C'est là une pratique, visant à gagner des clients, qui n'est pas étrangère à notre époque.

18. Dans notre corpus, il y a deux autres ouvrages qui portent le terme Arte dans leur titre: l'Arte de traducir el idioma Froncés al Castellano (1776) de Capmany et l'Arte de hablar bien frances (1781) de Chantreau. Nous ne pensons pas que le choix de ce terme par ces auteurs ait un rapport quelconque avec les conceptions de Billet. Il est bien plus probable qu'ils s'en servent suivant la distinction établie par y Encyclopédie, qui opposait grammaire générale -ou science des principes généraux de la parole écrite ou parlée dans toutes les langues- à grammaire particulière, ou art d'appliquer ces principes généraux à une langue particulière (Swiggers, 1984: 10; Stéfanini, 1994: 202; Lépinette, 1996: 164). 
19. La traduction des Métamorphoses d'Ovide faite par Isaac de Benserade n'est que mentionnée, mais non pas citée. Il y a également mention du Dictionnaire des rimes de La Noue, dont la première édition date de 1596, mais qui avait connu plusieurs rééditions au cours du $17^{\mathrm{e}}$ siècle.

20. Étant donné les qualités littéraires et chrétiennes qui, selon Buffier, ornaient les fables de Mlle Bernard, il a dû penser qu'un La Fontaine, menant une vie si peu exemplaire, ne méritait pas la moindre allusion.

21. En fait, à côté des grammaires proprement dites, on assiste au $18^{\prime}$ siècle à de nouvelles publications de Didlogos, c'est-à-dire d'ouvrages qui disposent en colonnes bilingues parallèles des conversations colloquiales. Les plus célèbres seront ceux de Sobrirto, publiés pour la première fois en 1708 et réédités tout au long du siècle. Sobrino, en fait, ne fait que prendre la relève des multiples rééditions des dialogues d'Oudin, lesquels, depuis 1604 , occupent tout le $17^{\mathrm{e}}$ siècle, étant liés, à leur tour, à la tradition du 16 e siècle (Lépinette, 1991: 141). La force de cette lignée dérive, dans une large mesure, de la conviction, partagée par beaucoup de grammairiens, que la syntaxe ne pouvait s'apprendre que par la pratique (Roldân Pérez, 1976: 216; Sânchez, 1987: 49; Caravolas, 1995: 301).

22. Ce texte est devenu une sorte de manuel de traduction dans l'enseignement du français langue étrangère et, par conséquent, l'un des textes de la littérature française les plus réputés. Novella, par exemple, dans sa grammaire de 1813, considère encore que cette œuvre tient dans la littérature française une place similaire à celle occupée par le Quichotte dans les lettres espagnoles: «Si tienes gusto en leer, / y deseas ilustrarte, / Conocer del nombre el arte, / Quál es su genio y poder: / El Tito Livio en Latin, / Metastasio en italiano, / El Quixote en castellano, / El Telémaco en francés, / y el Almeyda en portugués / Nunca dexes de la mano.» [Si tu as le goût de la lecture et que tu veuilles t'illustrer et connaître l'art, le génie et le pouvoir de l'homme, essaie d'avoir toujours sous la main, en latin, Tite-Live; en italien, Métastase; en espagnol, le Quichotte; en français, le Télémaque et, en portugais, Almeida]

23. Cette idée a pu être empruntée par Rousseau à Buffier, qui, comme lui, conseille le même genre de livres pour les débutants et mentionne aussi le nom de Bussy parmi les auteurs recommandés. Mais ce serait là la seule idée puisée par Rousseau chez le jésuite en ce qui concerne la méthode d'enseignement du français (comparez les pp. 62-74 de Rousseau, occupées par le chapitre XII «Del uso, y manejo de los libros», avec les pages 28-32 de Buffier (1711), occupées par le point «De la meilleure manière d'aprendre le François, \& quelque autre langue que ce soit»).

24. Cet ensemble nous semble très représentatif du goût théâtral du $18^{\mathrm{e}}$ siècle. De ce même point de vue, le tableau aurait été à peu près complet s'il y avait figuré le nom de Voltaire, comme ce sera le cas dans certaines rééditions postérieures (voir notre note 25). Mais la mention de Voltaire n'était certainement pas prudente dans l'Espagne de l'époque.

25. Cet ensemble sera considérablement refait dans les éditions de la grammaire de Chantreau parues au $19^{\mathrm{e}}$ siècle. Voir, par exemple, celle de 1811, qui déplace les fables de La Fontaine vers le groupe «Poésie» (où apparaissent également les Odes sacrées de Jean-Baptiste Rousseau), ajoute les œuvres de Voltaire et de Destouches à l'ensemble dramatique, et inclut dans le premier groupe les noms de Rollin, Fontenelle et Bitaubé.

26. Nous avons consulté pour faire cette étude pratiquement la totalité des grammaires de français à l'usage des Espagnols et des dictionnaires bilingues français-espagnols publiés au $18^{\mathrm{e}}$ siècle. Pour une raison de place, nous ne donnons ici que les références des œuvres -et des rééditions- auxquelles nous avons fait directement référence. On trouvera les références du reste de notre corpus dans Bruña Cuevas (1996a, 1998). Les sigles BN signifient «Bibliothèque nationale de Madrid». 1558 [1551]. Berlaimont, Noël de. Vocabulaire en quatre langues, Françoyse, Latine, Italienne, Espaignole. Louvain: Bartholomy de Grave. BN R/17916; 1604. Oudin, César. Dialogues en François \& Espagnol. Bruxelles: François Foppens. BN R/30490; 1673. Billet, Pierre-Paul. Gramática Francesa; dividida en dos partes [...] con un Arte Poética, o breve compendio de la Poesía Francesa. 
Saragosse: s.é. BN 3/47623; 1687? Gramática Francesa, dividida en dos partes. Anvers: H. \& C. Verdussen. BN 3/41273; 1688. Gramática Francesa, dividida en tres panes. Madrid: Bernardo de VillaDiego. BN 2/65893; 1708. Madrid: Juan García Infanzón. BN 3/19798; 1708. Sobrino, Francisco. Diálogos nuevos en Español y Francés. Bruxelles: F. Foppens. BN 3/24497; 1714. Vayrac, Jean de: El Arte Franges. [...] Con un Tratado de la Poesía. Paris: Pierre Witte. BN 3/31025-6; 1728. Torre y Ocón, Francisco de la. Nuevo Methodo breve, vtil, y necessario para aprender a escribir, entender, y pronunciar las dos principales Lenguas, Española, y Francesa. Madrid: luán de Ariztia. BN 3/20393 et R/36948; 1728-1731. El Maestro de las dos Lenguas. Diccionario Español, y Francés; Francés, y Español. Madrid: Juan de Ariztia. BN 3/50590-1; 1748. Galmace, Antoine. Llave nueva, y universal, para aprender con brevedad, y perfección la Lengua Francesa, sin auxilio de Maestro. Madrid: Gabriel Ramírez. BN 3/50840; 1754. Rousseau, Paul-François. Rudimentos de la Lengua Francesa, o Extracto de preceptos breves, y claros para su prompta inteligencia. Valladolid: Alonso del Riego. Bibliothèque de Santa Cruz de Valladolid 12682; 1763. Contaut, Pierre. Gramática Española y Francesa. Madrid: Imprenta del Diario. BN 5/1418; 1776. Capmany y Suris de Montpalau, Antonio de. Arte de traducir el idioma Francés al Castellano. Madrid: Antonio de Sancha. Nous avons consulté la réédition de María del Carmen Fernández Díaz (Saint-Jacques-de-Compostelle: Servicio de Publicaciones de la Universidad, 1987); 1786 [1781]. Chantreau, Pierre-Nicolas. Arte de hablar bien francés. Madrid: Antonio de Sancha. Bibliothèque universitaire de Séville 276/474; 1811. Lyon: Yvemault \& Cabin (J.M. Boursy). Bibliothèque des Lentes de Séville 4/862; 1790 [1789]. Gattel, Claude-Marie. Nouveau Dictionnaire Espagnol et François, François et Espagnol, avec Vinterprétation Latine de chaque mot. Lyon: Bruyset Frères. BN 5/4645-8; 1813. Pablo Antonio Novella: Nueva Gramática de la Lengua Francesa y Castellana. Alicante: Imprenta de España. BN 1/44617; 1815. Monfort, Luis. Principios de Lengua Francesa para uso de los Españoles. Valencia: Esteban. BN 1/33868.

\section{AUTEUR \\ MANUEL BRUÑA CUEVAS}

Université de Séville 